\title{
Social Intervention Impacts Action Anticipation, Goal Extraction, and Social Interest in Children With Autism Spectrum Disorder
}

\author{
Joshua L. Haworth ${ }^{1}$, Klaus Libertus ${ }^{2} \&$ Rebecca J. Landa ${ }^{3}$ \\ ${ }^{1}$ Science \& Learning Center, Department of Kinesiology, Whittier College, Whittier, CA, USA \\ ${ }^{2}$ Department of Psychology, University of Pittsburgh, Pittsburgh, PA, USA \\ ${ }^{3}$ Center for Autism and Related Disorders, Kennedy Krieger Institute, Baltimore, MD, USA \\ Correspondence: Dr. Rebecca J. Landa, Kennedy Krieger Institute, Center for Autism and Related Disorders, 3901 \\ Greenspring Ave, Baltimore, MD 21211, USA. E-mail: landa@kennedykrieger.org
}

Received: May 30, 2018 Accepted: June 29, 2018 Online Published: August 16, 2018

doi:10.5539/jedp.v8n2p95 URL: http://doi.org/10.5539/jedp.v8n2p95

\begin{abstract}
Anticipatory looking in the context of goal-directed actions emerges during the first year of life. However, children with autism spectrum disorder (ASD) often show diminished social gaze and anticipation while observing goal-directed actions. The current study examined a therapist-mediated social intervention targeting action-anticipation, goal-extraction, and social gaze in 18 children with ASD diagnosis. Before and after the intervention period, children viewed a video displaying a toddler repeatedly placing blocks into a bowl using a cross-body motion. Gaze to the actor's face and anticipatory gaze to the goal location were analyzed. Results revealed that young children with ASD understand repeated actions and demonstrate goal-extraction even before exposure to the intervention. Further, targeted social intervention experience led to a redistribution of attention in favor of the actor's face, while retaining action intention comprehension of the block transfer activity. Attention to social aspects during action observation by children with ASD could have favorable cascading effects on social reciprocity, social contingency, and theory of mind development.
\end{abstract}

Keywords: autism, action perception, anticipation, social cognition, intervention

\section{Introduction}

Development of social competence and collaboration requires an ability to infer meaning and intention of others' actions during dynamic human interaction. To make sense of others' action, children must integrate information from multiple sources including contextual, social, and action trajectory cues. From an early age, cognitive and attentional biases facilitate the development of abilities that support the formation of inferences about others' intentions (Legerstee, Anderson, \& Schaffer, 1998; Striano \& Reid, 2009). For example, the ability to anticipate others' goals based on their actions emerges during the first year of life (Gergely, Nádasdy, Csibra, \& Bíró, 1995; Gredebäck, Stasiewicz, Falck-Ytter, Rosander, \& von Hofsten, 2009; Falck-Ytter, 2010; Woodward 1998). Similarly, infants learn to form associations between facial cues, particularly gaze direction, and mental state (Scaife \& Bruner, 1975). These early-emerging abilities are foundational and support the development of a theory of mind and other social-cognitive skills (Senju, et al., 2010). For example, early coordinated joint engagement has been found to predict subsequent social and communication development (Adamson \& Bakeman, 1985; Adamson, 1995; Mundy \& Newell, 2007). Thus, early emerging impairments in children's ability to glean information about others' intentions could have cascading consequences for social development (Bhat, Landa, \& Galloway, 2011). This is of importance for children with autism spectrum disorders (ASD), who show social and communication delays later in life and have been noted to exhibit early emerging impairments in reciprocal social interaction (Landa, Holman, \& Garrett-Mayer, 2007; Wetherby, Watt, Morgan, \& Shumway, 2007).

Successful social interaction requires predicting the intentions and goals of other people's actions. Faces and limb movements provide critical cues for action anticipation (Vivanti et al., 2011) and both are mapped onto the same brain region (superior temporal sulcus; Puce, Allison, Bentin, Gore, \& McCarthy, 1998; Allison, Puce, \& McCarthy, 2000; Servos, Osu, Santi, \& Kawato, 2002). Specifically, the directions of gaze and head orientation provide referential cues that permit prediction about the focus of a person's attention (Phillips, Wellman, \& Spelke, 2002). Eye gaze also elicits a response in the observer to look in the same direction (Gibson \& Pick, 1963) 
resulting in a shared focus of attention, known as joint attention. Similarly, seeing the start of someone's reaching movement permits prediction of the intention behind their action. For example, upon seeing someone reach for a cup of coffee, the viewer may predict the person's intention to drink (Frith \& Frith, 2006). Indeed, typically developing individuals demonstrate anticipatory gaze to the target object of a person's reach (Flanagan \& Johansson, 2003). Use of such facial and action cues permit children to predict others' intentions and affords them the ability to respond contingently and to establish joint attention, which supports interpersonal synchrony.

During everyday interactions, facial cues contribute to the interpretation of others' action intention. However, children with ASD often exhibit atypical attention to faces and may not make full use of facial cues. For example, the attention bias for faces that characterizes young children with typical development is not characteristic of young children with ASD (Chawarska, Volkmar, \& Klin, 2010). Children with ASD also demonstrate atypical attention allocation to specific facial regions (Chawarska \& Shic, 2009), and overall slower face processing (McPartland, Dawson, Webb, Panagiotides, \& Carver, 2004). Emerging in infancy (Chawarska et al., 2010), atypical face processing may interfere with neural development supporting the formation of mental representations of social intentionality and events (Klin, Jones, Schultz, \& Volkmar, 2003).

As early as two years of age, children with ASD exhibit disrupted preferential attention to human biological motion (Klin, Lin, Gorrindo, Ramsay, \& Jones, 2009; Kaiser \& Pelphrey, 2012). Evidence regarding understanding intentions associated with others' actions in children with ASD is mixed. On the one hand, children with ASD do not seem to appreciate others' intentions as they show delayed learning to imitate others' actions (Rogers, Hepburn, Stackhouse, \& Wehner, 2003) but imitate 'accidental' (i.e., unintentional) acts (D'Entremont \& Yazbek, 2007). On the other hand, no differences in intention comprehension (e.g., imitating rational more often than non-rational acts) between children with autism and typical development have been reported (Hamilton, Brindley, \& Frith, 2007; Rogers, Young, Cook, Giolzetti, \& Ozonoff, 2010). Imitation paradigms have relatively high task requirements (i.e., a gross motor response) that may interfere with assessing performance in children with ASD. Using eye-tracking paradigms can address this limitation by examining attentional characteristics fundamental to social comprehension (e.g., eye movements) without the need for peripheral gross motor responses.

Eye tracking paradigms have been used to examine action anticipation in children with ASD (Falck-Ytter, 2010; Vivanti, et al., 2011). Results of these studies show that school-age children with ASD produce anticipatory eye movements when observing a person repeatedly moving objects from one side of a table into a container on the other side (Falck-Ytter, 2010). Similarly, high functioning children and adolescents with ASD accurately predicted an actress's intentions based on her actions on objects, but not from her directional head turn cues alone (Vivanti, et al., 2011). However, children with ASD failed to shift their gaze from the actress's hands to her head while viewing the scene - unlike their neurotypically developing peers. Thus, children with ASD do not appear to integrate information from the face/head region with information from the hands to infer others' intentions. It remains unknown whether children with ASD extract the intended goal of the behavior, and thus show quicker anticipatory gaze on subsequent trials of the same viewing condition. Extracting the goal of an observed action (referred to as "goal extraction") is critical for engaging in the contingent, reciprocal behavior that is needed to achieve interpersonal synchrony during interactions (Flanagan, Rotman, Reichelt, \& Johansson, 2013).

In summary, previous findings indicate that children with ASD have persistent difficulty in attending to and interpreting non-verbal cues about others' goals and intentions. Early intervention can improve social outcomes in young children with ASD (Kasari, Gulsrud, Wong, Kwon, \& Locke, 2010; Landa, Holman, O'Neill, \& Stuart, 2011). However, no study to date has examined the effects of early intervention on attention allocation in children with ASD to socially relevant information during observation of goal-directed actions. The current study aims to close this gap in the literature and examines overt visual attention during observation of an ecologically valid action sequence (i.e., the sequence includes several social cues, but not all are relevant to the ongoing action). Closing this gap is of considerable practical importance, especially given that one in 59 children is affected with ASD (Baio et al., 2018). Based on reports of older children with ASD, we hypothesized that young children with ASD would show difficulty extracting the goal of an action over repeated performances. We further hypothesized that an intervention targeting attention to faces, joint attention, imitation, and communication would lead to increased social interest and goal extraction during action observation without perturbing action anticipation. If this hypothesis is confirmed, a major stride toward establishing interventions that ameliorate the effects of ASD will be taken. The implementation of such interventions, early in life during a formative period of neurodevelopment, could establish more efficient social learning pathways and cascading effects that support ever-increasing social and communicative competence in children with ASD. Long-term effects could expand social opportunities and success for individuals with ASD. 


\section{Methods}

The Johns Hopkins Institutional Review Board approved all procedures of this study. The families of all eligible children gave written informed consent for their own and their child's participation. The research was registered with clinicaltrials.gov under the trial number NCT01425918 and all methods or measures remained unchanged after trial commencement.

\subsection{Participants}

The final sample of this randomized controlled trial (RCT) consisted of 18 children with prior ASD diagnosis (see Table 1). A total of 110 children were recruited for the RCT between March, 2012 and March, 2014. Of these children, 86 met eligibility criteria and were randomized into a caregiver-training only $\left(\mathrm{C}_{0}\right)$ or combined caregiver-training plus classroom-based $\left(\mathrm{C}_{+}\right)$intervention group. From the eligible children, 25 families $\left(13 \mathrm{C}_{0}, 12\right.$ $\left.\mathrm{C}_{+}\right)$declined participation and another 7 children $\left(6 \mathrm{C}_{0}, 1 \mathrm{C}_{+}\right)$did not complete the treatment protocol. A total of 54 children completed the study. Of those children, 10 in the $\mathrm{C}_{0}\left(3\right.$ female) and eight in the $\mathrm{C}_{+}(3$ female) group had usable eye tracking data ( $>25 \%$ samples acquired) during both the pre- and post-intervention assessments. Data from these 18 participants was used to examine anticipatory looking and goal extraction in young children with ASD before intervention, and to examine the malleability of these functions following an early intervention targeting social development. See Figure 1 for an enrollment CONSORT flow diagram.

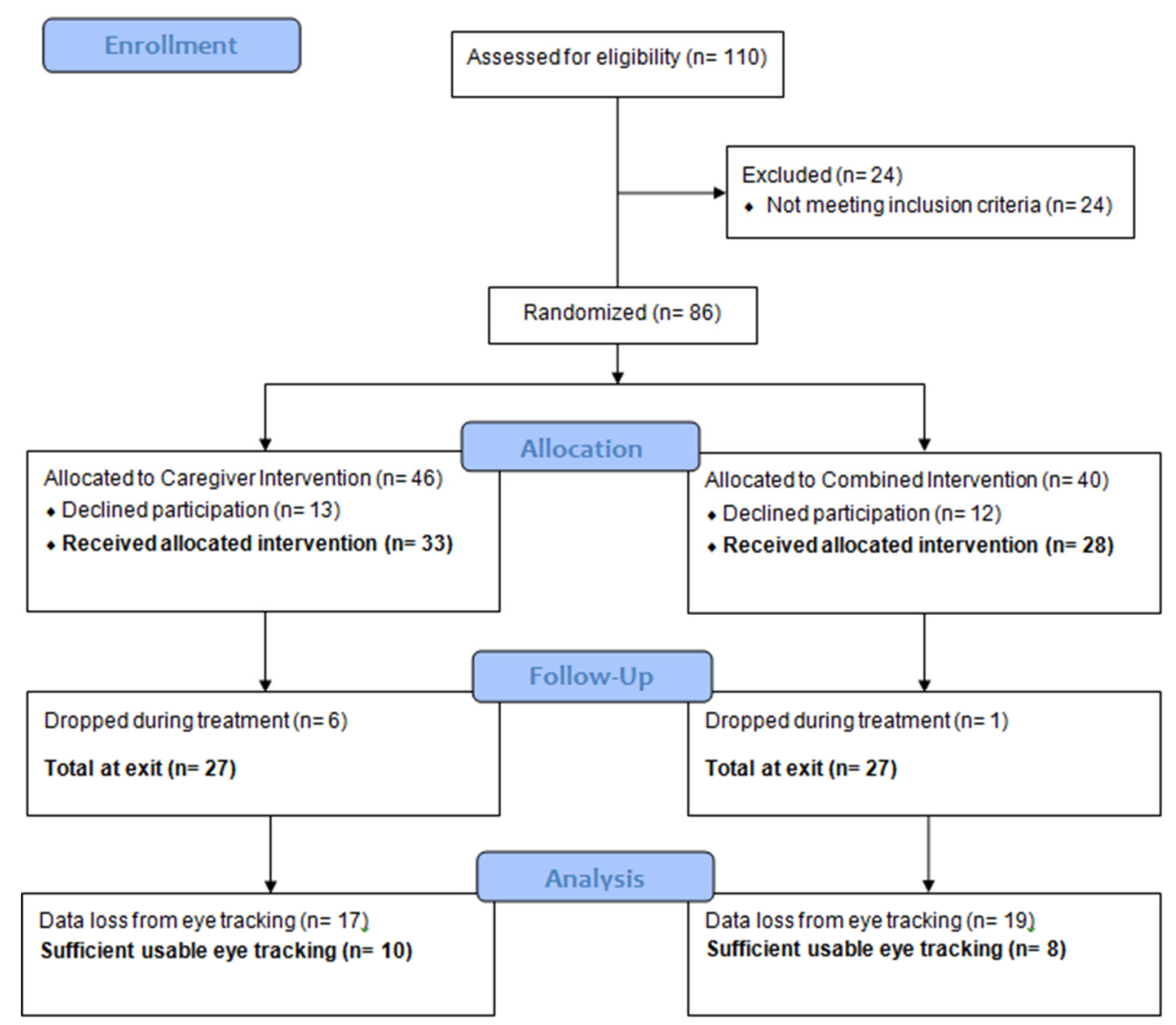

Figure 1. Consort diagram, detailing initial recruitment, randomization, and final sample for the current study

Families were recruited through autism advocacy organizations, ASD intervention programs, and community events. Inclusion criteria included: 24 to 42 months of age; English as primary language; meeting criteria for ASD or autism on the Autism Diagnostic Observation Schedule-Generic (ADOS; Lord, Rutter, DiLavore, \& Risi, 2002; Lord, Luyster, Gotham, \& Guthrie, 2012) and having an expert clinical judgment of ASD. Exclusionary criteria included: severe birth trauma or defects; head injury; prenatal illicit drug or excessive alcohol exposure; being a foster child or adopted; and known genetic disorders conferring ASD risk. Table 1 summarizes the demographic 
characteristics of the 18 children and their participating caregivers. There were no significant differences between groups on any measured demographic variables ( $p s>.05$, independent-samples t-tests), including on the Mullen Scales of Early Learning (MSEL; Mullen, 1995) Early Learning Composite (ELC).

Table 1. Participant demographics

\begin{tabular}{|c|c|c|c|c|}
\hline \multirow{3}{*}{ Measure } & \multicolumn{4}{|c|}{ Training Group } \\
\hline & \multicolumn{2}{|c|}{ Combined $\left(\mathrm{C}_{+}\right)$} & \multicolumn{2}{|c|}{ Caregiver Only $\left(\mathrm{C}_{0}\right)$} \\
\hline & \multicolumn{2}{|l|}{$\mathrm{n}=8$} & \multicolumn{2}{|c|}{$\mathrm{n}=10$} \\
\hline Caucasian & \multicolumn{2}{|l|}{4} & \multicolumn{2}{|l|}{2} \\
\hline African American & \multicolumn{2}{|l|}{0} & \multicolumn{2}{|l|}{5} \\
\hline Asian & \multicolumn{2}{|l|}{0} & \multicolumn{2}{|l|}{1} \\
\hline Hispanic & \multicolumn{2}{|l|}{2} & \multicolumn{2}{|l|}{0} \\
\hline \multirow[t]{2}{*}{ Multi-racial } & \multicolumn{2}{|l|}{2} & \multicolumn{2}{|l|}{2} \\
\hline & $\overline{\mathrm{x}}$ & $S D$ & $\overline{\mathrm{x}}$ & $S D$ \\
\hline Age (months) & 32.1 & 4.3 & 34.2 & 6.0 \\
\hline Hollingshead & 53.1 & 10.0 & 49.4 & 14.3 \\
\hline Mullen ELC & 66.5 & 15.2 & 57.7 & 8.2 \\
\hline \multicolumn{5}{|l|}{ ADOS CSS } \\
\hline pre & 6.3 & 0.7 & 6.6 & 2.1 \\
\hline post & 5.8 & 0.9 & 6.2 & 1.8 \\
\hline \multicolumn{5}{|c|}{ Child intervention dosage (hours/week) } \\
\hline Caregiver training & 8.3 & 6.2 & 7.7 & 6.9 \\
\hline Classroom intervention & 8.3 & 0.4 & na & $\mathrm{Na}$ \\
\hline
\end{tabular}

Note. ${ }^{*}$ ELC = Early Learning Composite, ADOS CSS = Autism Diagnostic Observation Schedule Calibrated Severity Score.

\subsection{Intervention Procedures}

\subsubsection{Caregiver Training $\left(\mathrm{C}_{0}\right.$ and $\left.\mathrm{C}_{+}\right)$}

Caregivers in both the $\mathrm{C}_{0}$ and $\mathrm{C}_{+}$groups attended weekly two-hour center-based Caregiver Training workshops for five months. To prevent contamination, caregivers from the $\mathrm{C}_{0}$ and $\mathrm{C}_{+}$groups attended separate workshops. Each cohort consisted of up to six primary caregivers of children with ASD. The curriculum focused on implementation of child-responsive engagement strategies (Maloney, 2007) and joint action routines to promote children's social interaction, communication, and play development. Training was standardized across both groups and delivered using intervention materials including PowerPoint presentations, illustrative video examples, home implementation planning, practice activities and information sheets, role-playing, and sharing reflections about experiences implementing the strategies at home. While no direct coaching of caregiver implementation with their child was provided, troubleshooting discussions were held each session to identify action plans for refinements in caregiver implementation of the intervention strategies based on caregivers' descriptions of their experiences and perceived barriers to child engagement. Caregivers were expected to practice the strategies daily at home, which was monitored via daily caregiver implementation diary (no group difference; see Table 1).

\subsubsection{Classroom-Based Intervention $\left(\mathrm{C}_{+}\right)$}

For children of parents in the $\mathrm{C}_{+}$group, a manualized, center-based, classroom-based group intervention (Landa, Holman, O'Neill, \& Stuart, 2011) was delivered 2.5 hours/day, four days/week, for five months (average 83\% attendance rate; see Table 1). Naturalistic behavioral intervention strategies (Schreibman et al., 2015) were used to promote children's understandings of bi-directional influences involving self and peers (social contingencies). Class size ranged from three to six children; with three interventionists. Intervention targets included: (a) face processing (attention to faces, face recognition), (b) action understanding (attention to others' socially-relevant actions, anticipation and preparedness for response to others' actions), (c) imitation (recognition of being imitated, 
imitation of others), and (d) communication (language comprehension and expression, gesture production). Instructional activities targeting social development included: constructing faces, completing face-manipulation activities, engaging in joint attention communication activities, engaging in supported reciprocal social interactions, and engaging in imitation acts with and without objects. The lead interventionists achieved at least $80 \%$ fidelity.

Before onset (baseline) and after completion (post) of the 5-month intervention period, trained clinical researchers assessed all children in both conditions, with the eye-tracking measure reported here being a pre-specified secondary outcome measure.

\subsection{Eye Tracking Task}

\subsubsection{Apparatus}

Before and after treatment, children from both groups participated in an eye-tracking task designed to assess action anticipation, goal extraction, and social interest. Children were seated in a dimly lit room in front of a 22 " computer monitor $(43.60 \times 28.1$ degrees of visual angle, with a resolution of $1680 \mathrm{x} 1050$ pixel $)$ and a Tobii X-120 eye tracker placed at a distance of $60 \mathrm{~cm}$ from the participants. Tobii Studio was used for calibration (using a 9-point fixation procedure), stimulus presentation, and data collection. No instructions were provided to the children regarding how they should attend to the videos.

\subsubsection{Stimulus}

The stimulus consisted of a video-sequence showing a social agent (15-month-old toddler) moving two blocks (one-at-a-time) into a clear bowl using a cross-body motion (see Figure 2). During movement of the first block, the actor looked at the bowl immediately upon touching the block. For the second block, the actor briefly glanced to his right (away from the target bowl) immediately after touching the block and then oriented his attention to the bowl halfway through the event. The rightward glance is representative of noise in the social cues that are present during ecologically typical behavior and was not intended to serve as manipulation of the viewer's behavior. The time interval between initial contact of the actor's hand on the block and its arrival at the target bowl was $1170 \mathrm{~ms}$ and 1720ms for trials one and two, respectively. Research assistants conducting the eye tracking assessment were blinded to children's group assignment.

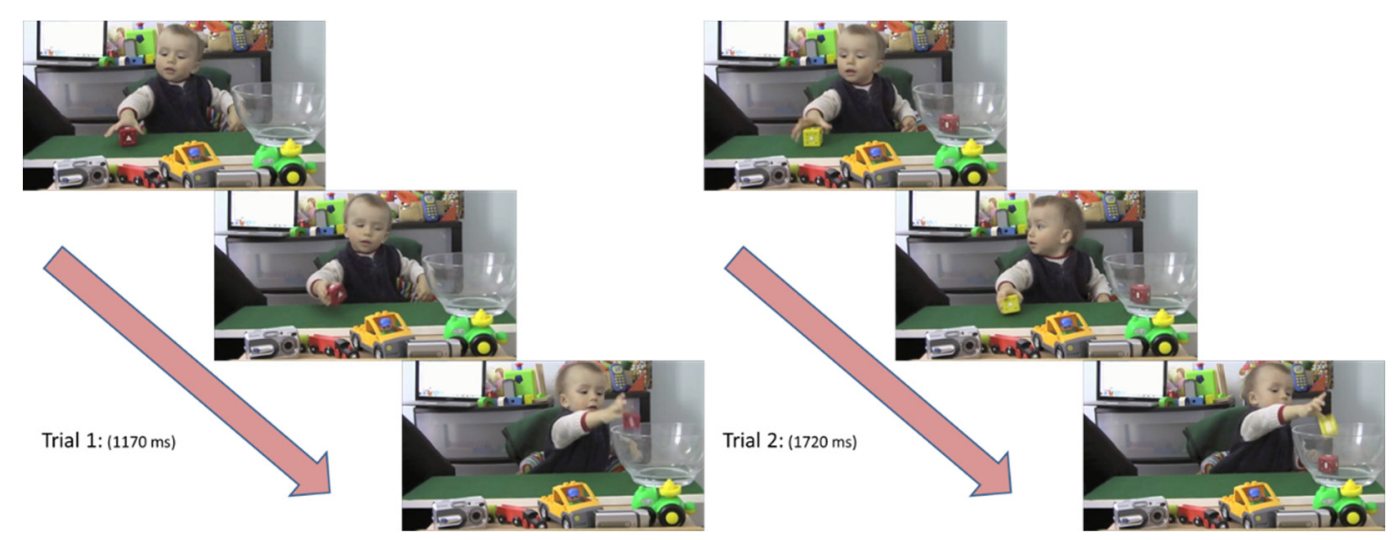

Figure 2. Illustration of trials one and two from the action observation task

\subsubsection{Dependent Measures}

Raw data were exported and processed with custom Matlab code (The Mathworks, MA). Areas of interest (AOI) were defined by rectangles around the face (360 x 290 pixel), hand (360 x 260 pixel), and the target bowl (360 x 260 pixel). Three main dependent measures were examined in the current study: action anticipation, goal extraction, and AOI look time. 'Action anticipation' was defined as the arrival of the participant's gaze at the target location relative to the placement of the block at this location in the video (with negative values indication anticipation, and positive values indicating reaction). 'Goal extraction' was defined as the difference in action anticipation time between the first and second trials (action anticipation $\mathrm{T}_{2}-$ action anticipation $_{\mathrm{T} 1}$ ). Larger values indicate faster looks towards the bowl on the second trial and successful goal extraction. Finally, 'social interest' was defined as total looking durations towards the face (social), 'non-social interest' was defined as looking 
durations towards the hand (non-social) AOIs. Durations were expressed as the proportion of the gaze dwell time within the AOI, relative to the duration of the video sequence. 'Pre- to post-intervention ratio of AOI looks' also were calculated separately for the hand and face AOIs; defined as the AOI look time at post-intervention divided by the AOI look time at pre-intervention (Post/Pre, reported as \%). These values were centered at $100 \%$ (by subtracting $100 \%$ from the calculated ratios, as a value of $100 \%$ would indicate no change in behavior from pre- to post- intervention) in order to provide directional rate changes. Positive change values indicate increased interest to the particular AOI after intervention experience, and negative values indicate decreased interest.

\subsection{Statistical Approach}

All statistical manipulations and tests were performed using SPSS (v.22). Preliminary analyses examined all variables and confirmed heterogeneity of variances, normal distribution, and absence of outliers in all but one variable. The Parent-Only-Hand-AOI group showed deviations from normality (Shapiro-Wilk $p=.002$ ). This variable was analyzed in a repeated measures ANOVA, which is robust to violations of normality.

Action anticipation times were measured in response to a behavior lasting fewer than $2 \mathrm{~s}$ and resulted in some missing data (e.g., child looking away, blinking, or not anticipating event). From our sample of 18 children, 10 showed one missing trial for this analysis. To account for missing data points, multiple imputation was employed to generate ten surrogate sets from the measured data. This approach is robust to resolve up to $50 \%$ missing data (Schafer, 1999). Imputed values are random draws from the posterior predictive distribution of the missing data, given the observed data. After imputing the data sets, conventional statistical estimation methods were applied to each of the ten resulting data sets. Pooled means (M) and standard error values (SE) are reported, descriptive of the set of imputed datasets. For inferences, the average p-values $(\overline{\mathrm{p}})$ and means of effect size (Cohen's d, $\bar{d}$; or partial eta-squared, $\overline{\eta_{p}^{2}}$ ) from the set of tests of each imputed dataset are reported. For each hypothesis test conducted in this study, all 10 of the imputed datasets corroborated their results, meaning statistical results were consistent across each of the 10 imputed datasets.

In contrast to action anticipation, AOI look time measures spanned several seconds and thus were less susceptible to causes of missing data. In all reported cases, the child's gaze was successfully recorded for at least $25 \%$ of the duration of the trials. Thus, no imputation of these values was needed for our measure of "social interest."

Analyses on pre-intervention data combined children from both groups to provide insight about action anticipation and goal extraction in young children with ASD. A single-sample t-test was used to assess whether children with ASD spontaneously exhibited anticipatory looking behavior by comparing the first trial action anticipation times against zero (i.e., no anticipatory look). A paired t-test was used to assess goal extraction by comparing times on trials 1 and 2 (to determine if a difference exists). Analyses of intervention effects focused on action anticipation, goal extraction, and social interest (looking durations to face and hand AOIs). A paired t-test was used to assess change in action anticipation on the first trial at pre- and post- intervention. A Group (2) by Time (2) repeated measures ANOVA was used to assess the impact of the intervention on the expressed goal extraction behavior by comparing the goal extraction times (difference in action anticipation times, on trials 1 and 2), between groups, at both pre- and post-intervention. A separate Group (2) by AOI (2) repeated measures ANOVA was used to compare social interest, represented by the pre- to post-intervention ratio of AOI looks, between groups, for both face and hand AOIs.

\section{Results}

\subsection{Pre-Intervention: Action Anticipation and Goal Extraction}

To examine whether young children with ASD spontaneously exhibit anticipatory looking behavior before receipt of our intervention conditions, we conducted a single-sample t-test comparing action anticipation time during the first trial to a value of zero (which would indicate no anticipatory look). Results indicate no difference from zero $(M=126.98 \mathrm{~ms}, S E=115.56 \mathrm{~ms}, t(17)=1.1, \bar{p}=.287, \bar{d}=.259)$, suggesting the children in our study did not show spontaneous anticipatory looking behavior. However, some researchers have proposed a more liberal latency value of $200 \mathrm{~ms}$ rather than 0 to account for oculomotor coordination time (Gredebäck, et al., 2009; Canfield, et al., 1997; von Hofsten, Uhlig, Adell, \& Kochukhova, 2009). Using this more liberal latency value, our results support the presence of action anticipation $(M=326.98 \mathrm{~ms}, S E=115.56 \mathrm{~ms}, t(17)=2.8, \bar{p}=.012, \bar{d}=.667)$. This agrees with previous studies using similar analyses to report spontaneous action anticipation in children with ASD (Falck-Ytter, 2010).

To examine goal extraction, a paired t-test compared action anticipation on the first and second trials. Results indicate goal extraction took place (increased anticipatory looking behavior), looking sooner to the bowl (relative to block arrival) on the second trial than on the first $\left(M_{\text {diff }}=784.01 \mathrm{~ms}, S E=198.14 \mathrm{~ms}, t(17)=3.98, \bar{p}<.002, \bar{d}\right.$ 
$=.933$ ). These findings refute our hypothesis that young children with ASD would have difficulty demonstrating goal extraction when observing repeated goal-directed actions prior to receiving an intervention targeting social cognition and communication.

\subsection{Treatment Effects on Looking Behavior}

To test whether intervention impacted action anticipation, a paired t-test compared trial one performance at preand post-intervention. Results indicate no change in action anticipation after intervention $\left(M_{\text {diff }}=120.19 \mathrm{~ms}, S E=\right.$ $178.85 \mathrm{~ms}, t(17)=.674, \bar{p}=.517, \bar{d}=.159)$. A Group $\left(\mathrm{C}_{0}\right.$ versus $\left.\mathrm{C}_{+}\right)$by Time (pre- versus post-intervention) repeated measures ANOVA was conducted to examine the effect of the intervention on goal extraction behavior (see Figure 3). Results revealed no main effect of Group or Time, but a significant Group by Time interaction, $\bar{F}(1,16)=9.07, \bar{p}=.011, \overline{\eta_{p}^{2}}=.358$. Simple main effects within Group showed a significant decrease in goal extraction in the $\mathrm{C}_{+}$group $\left(\bar{p}=.010, \overline{\eta_{p}^{2}}=.370\right)$, but no change in the $\mathrm{C}_{0}$ group $(\bar{p}=.335)$. Simple main effects within Time indicate that the $\mathrm{C}_{0}$ and $\mathrm{C}_{+}$groups did not differ in goal extraction at pre-intervention. However, at post-intervention, the $\mathrm{C}_{+}$group demonstrated a significantly smaller goal extraction score than the $\mathrm{C}_{0}$ group $(\bar{p}$ $=.008, \overline{\eta_{p}^{2}}=.376$ ). While children in the $\mathrm{C}_{+}$group continue to show goal extraction following treatment, these results suggest that their gaze arrives at the target bowl closer to the arrival of the actor's hand than it did prior to treatment. This change is likely due to the children in the $\mathrm{C}_{+}$group gazing more at other elements of the scene at the post-intervention measurement. The social interest analyses will explore this possibility.

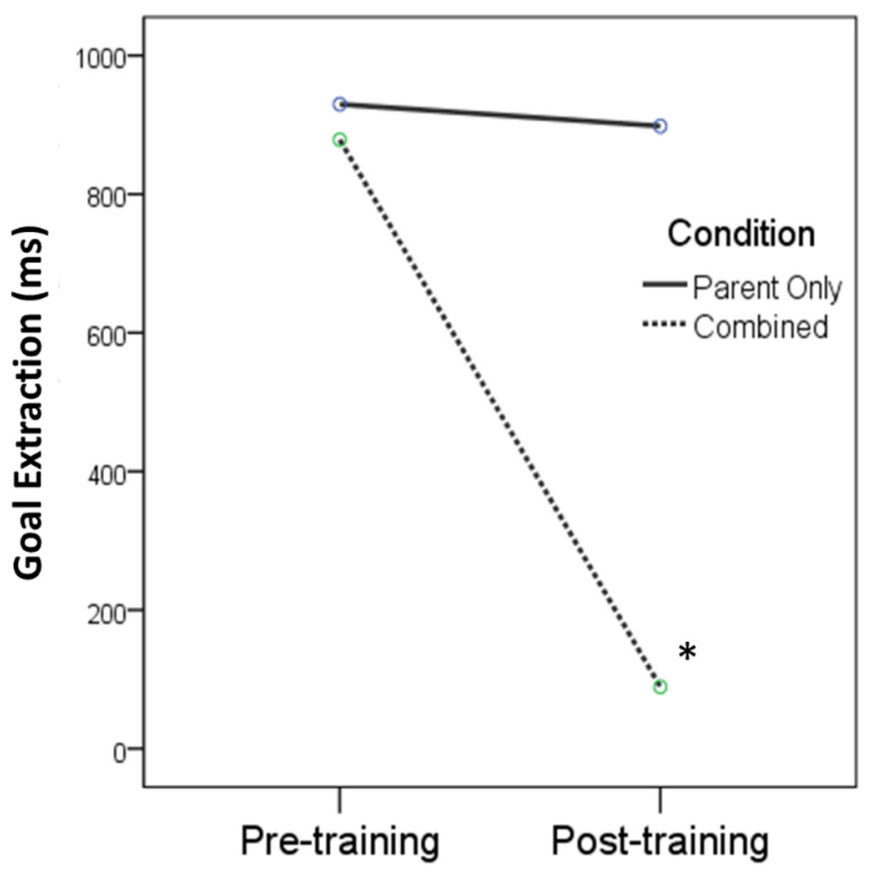

Figure 3. Goal extraction results before and after intervention by condition

$* \mathrm{p}<.05$ for within- and between-group comparisons.

\subsection{Social Interest}

A Group $\left(\mathrm{C}_{0}\right.$ versus $\left.\mathrm{C}_{+}\right)$by AOI (Face versus Hand) repeated measures ANOVA was used to assess intervention effects on attention allocation to social information in the naturalistic dynamic scene. This analysis compares the separate pre- to post-intervention ratio of AOI looks to the face and hands (see Figure 4). No main effect of Group or AOI was identified, but a significant Group by AOI interaction, $F(1,16)=6.46, p=.022, \eta_{p}^{2}=.288$, was identified. Simple main effects within Group show a marginal difference of AOI look time change with the $\mathrm{C}_{0}$ group looking more to the hand than to the face $\left(p=.054, \eta_{p}^{2}=.212\right)$. Thus, the $\mathrm{C}_{0}$ group tended to show an increased focus from pre- to post-intervention toward mechanical information afforded by watching hand motion rather than the social information present in the face. Simple main effects within AOI indicate groups did not differ on the pre- to post-intervention ratio of $\mathrm{AOI}$ looks to the hand region. However, the $\mathrm{C}_{+}$group had a larger pre- to 
post-intervention increase in the ratio of AOI look to the face than the $\mathrm{C}_{0}$ group $\left(p=.016, \eta_{p}^{2}=.313\right)$. Together, these results indicate that the $\mathrm{C}_{+}$group showed increased interest to social information after intervention.

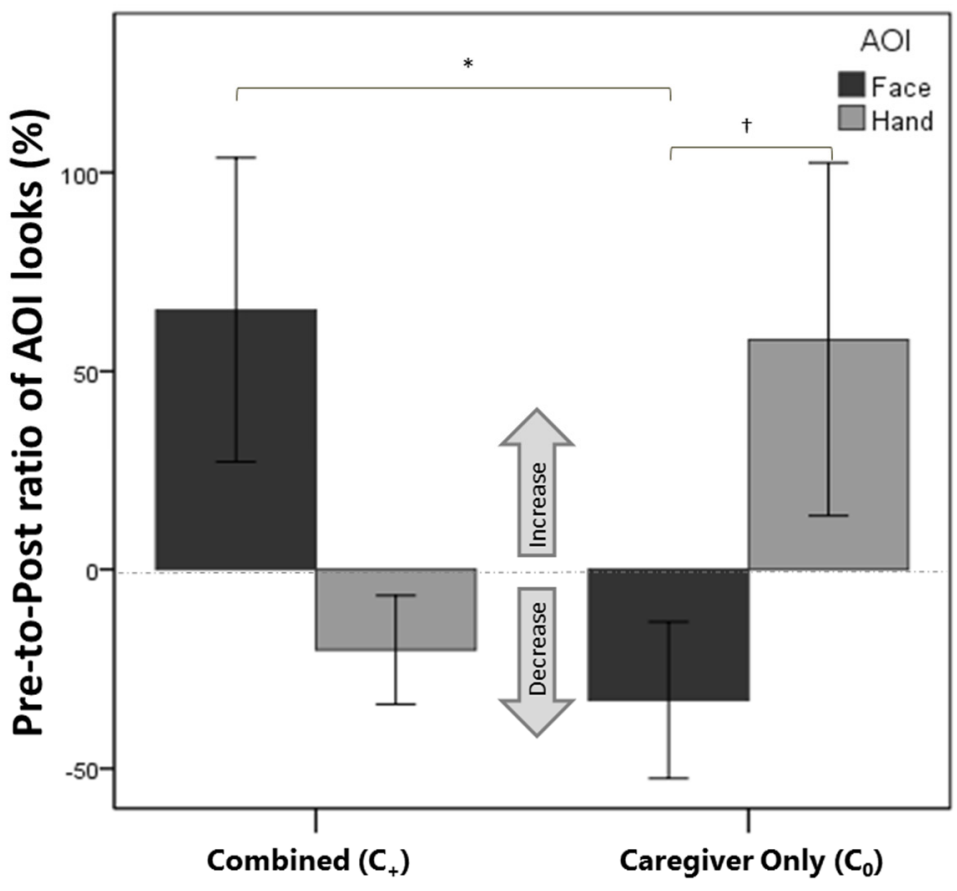

Figure 4. Ratio of the proportion of looks to face and hand areas of interest (AOI) from pre- to post-intervention. Positive values indicate increased attention to the AOI following intervention; negative values indicate a decrease $* p<.05, \dagger p<.10$.

\section{Discussion}

The current study investigated action anticipation, goal extraction, and social interest in young children with ASD while viewing a naturalistic scene of another child engaging with toy blocks. Further, we examined the effects of a classroom-based intervention tailored to promote social engagement and communication on children's social attention during action observation.

\subsection{Pre-Intervention: Action Anticipation and Goal Extraction}

Before intervention, we examined two aspects of action intention comprehension in children with ASD: action anticipation and goal extraction. Our results suggest that 2- to 3-year-old children with ASD demonstrate action anticipation when a lenient anticipation threshold is used. Children's gaze arrived at the target about $127 \mathrm{~ms}$ before the block arrived at this location, significantly earlier than the lenient threshold of $200 \mathrm{~ms}$ after block arrival. This finding supports previous findings where five-year-olds with ASD accurately predicted action outcomes when viewing another's actions on objects (Falck-Ytter, 2010). However, please note that our results do not show action anticipation when using a more stringent definition of anticipation that compares arrival of gaze to the actual arrival of the block at the target ( $0 \mathrm{~ms})$.

Further, analyzing the difference between the first and second trial action anticipation times, which represents the construct of goal extraction, we found that our participants' anticipatory eye gaze was more than half a second faster on the second trial compared to the first trial. This confirms that children were able to predict the goal-directed action outcome better after just one prior exposure to the behavior. This finding indicates that young children with ASD benefit from trial-to-trial learning when observing the actions of another young child (here a toddler) as opposed to an adult (as in Vivanti, 2011). Observation and interpretation of actions performed by a peer rather than an adult represent an understudied area but are of great importance given typically developing children's strong interests in dynamic interaction with other children. 


\subsection{Treatment Effects on Looking Behavior and Social Interest}

To better understand the distribution of interest to social and non-social aspects, we examined allocation of attention to the face and hand regions of the actor during model viewing pre- and post-intervention. Post-intervention, the $\mathrm{C}_{0}$ group showed a trend toward greater allocation of attention to the actor's hand with a concurrent decrease in attention to the face. In contrast, the $\mathrm{C}_{+}$group exhibited an increased allocation of attention to the actor's face while maintaining certain aspects of action intention comprehension (goal extraction). Overall, these findings indicate that young children with ASD receiving a targeted social classroom-based (group) intervention make gains in social attention and, perhaps, understanding. Further research is needed to determine whether, without such targeted intervention delivered with consistency by a trained interventionist over the course of multiple months (here, five months) during early development, attention biases to mechanistic features become greater in young children with ASD.

Understanding others' actions is a multi-faceted process that requires integration of contextual, social, and mechanical information. This integrative process may be impaired in children with ASD, who may focus more on the action (or mechanical information) at the expense of focusing on the context or social information in the scene. Vivanti and colleagues (2014) reported that children with ASD showed lower levels of goal monitoring and responsiveness to social cues compared to a matched group of children with typical development. Similarly, children with ASD exhibited difficulty inferring action intentions when gaze cues were pivotal in determining the course of the action, and generally looked less at the face of the model demonstrating the action to be imitated (Vivanti et al., 2011). Other reports suggest that children with ASD, unlike those without ASD, imitate 'accidental' actions of a person without showing appreciation of the person's intentions (D'Entremont, 2007; Meltzoff \& Brokks, 2001). Despite this presumed difficulty integrating mechanical and social cues to infer the intentions of observed actions, we found that young children with ASD, prior to receiving intervention, showed evidence of goal extraction but may have relied mainly on the mechanistic feature of the observed action during this process.

Following intervention, those children receiving targeted social and communication training $\left(\mathrm{C}_{+}\right.$group $)$developed a different pattern of attention allocation than children not receiving this intervention $\left(\mathrm{C}_{0}\right.$ group). Specifically, children in the $\mathrm{C}_{+}$group have increased proportion of time looking at the actor's face following intervention, while retaining goal extraction. This may be interpreted as an improvement in overall attention allocation during observation of complex social scenes by integrating the distributed social cues about a person's action intention. Such integration may permit greater semantic encoding of observed human action and, thus, greater action understanding as opposed to mainly mechanically-based action anticipation. Further, it has been noted that distributing attention across an interaction partner's hand actions and face may contribute to the development of joint attention in children (Yu \& Smith, 2017). Indeed, the $\mathrm{C}_{+}$group's post-treatment gaze performance more closely resembles patterns observed in typically developing children (Vivanti, 2011; Carpenter \& Tomasello, 1995; Castiell, 2003; Hobson \& Hobson, 2007). Children in the $\mathrm{C}_{+}$group actively participated in high dosage, strategic therapeutic activities focused on attention to, and gaining information from, faces. Most such activities involved naturalistic social engagement that included peer interaction. Thus, targeted social learning experiences appear to have had a major impact on the process of social attention and action observation and understanding, possibly laying the foundation for improved theory of mind abilities later in development. Despite the training that parents received to engage with their children in face-to-face, developmentally appropriate and child-contingent ways, the focused peer-to-peer engagement fostered by a trained interventionist in a classroom-based group early intervention appears to have served as ingredients activating a shift in social attention.

One potential limitation of the present study is the sample size. Despite the sample size, the novel data presented herein offer promise of malleability in a core deficit of autism when specifically targeted in intervention at an early age. This study requires replication in order to obtain confidence in generalizability to other young children with ASD. In addition, examining behavioral data focused on synchrony of social interaction of the children in this study could add ecological validity to the biological eye tracking data presented herein. Future research is needed to replicate and extend the present findings. Both single case design and randomized controlled trials would be beneficial in examining malleable and pivotal intervention targets, such as the ones mentioned in the present study, in early intervention for children with ASD. Research also is needed to understand whether and what type of scaffolded experiences young children with ASD require to sustain and further capitalize on improvements of the nature reported herein.

\section{Conclusion}

The current findings demonstrate malleability of social-cognitive development in young children with ASD in response to early social-focused group intervention delivered by a trained interventionist. Both groups studied here 
showed evidence for goal extraction during viewing of repeated goal-directed actions - even before intervention. However, after intervention, the groups differed in attention distribution across hand and face areas in the social scene. The group receiving the supplemental classroom-intervention progressed from allocating attention to the mechanical aspects of the action before intervention to looking more at the socially relevant aspects of the scene (i.e., the actor's face) after the intervention. This suggests that a group classroom-based social intervention, delivered by professionals and paraprofessionals, focusing on social interest and engagement facilitates the integration of social information during processing of dynamic scenes beyond that of a caregiver-only education intervention. Such integration of attention by children with ASD to social aspects during an action routine at a young age could have favorable cascading effects on social reciprocity, joint attention, and theory of mind development.

\section{References}

Adamson, L., \& Bakeman, R. (1985). Affect and attention: Infants observed with mothers and peers. Child Development, 56, 582-593. https://doi.org/10.2307/1129748

Adamson, L. B. (1995). Joint attention, affect, and culture. In C. Moore \& P. J. Dunham (Eds.), Joint attention: Its origins and role in development (pp. 205-221). Hillsdale, NJ: Erlbaum.

Allison, T., Puce, A., \& McCarthy, G. (2000). Social perception from visual cues: role of the STS region. Trends in Cognitive Sciences, 4(7), 267-278. https://doi.org/10.1016/S1364-6613(00)01501-1

American Psychiatric Association. (2013). Diagnostic and statistical manual of mental disorders (DSM-5). Washington, DC: American Psychiatric Association. https://doi.org/10.1176/appi.books. 9780890425596

Bhat, A. N., Landa, R. J., \& Galloway, J. C. C. (2011). Current perspectives on motor functioning in infants, children, and adults with autism spectrum disorders. Physical Therapy, 91(7), 1116-1129. https://doi.org/10.2522/ptj.20100294

Baio, J., Wiggins, L., Christensen, D. L., Maenner, M. J., Daniels, J., Warren, Z., ... Dowling, N. F. (2018). Prevalence of autism spectrum disorder among children aged 8 years. Autism and Summary, 67(6), 1-23. https://doi.org/10.15585/mmwr.ss6706a1

Canfield, R. L., Smith, E. G., Brezsnyak, M. P., Snow, K. L., Aslin, R. N., Haith, M. M., ... Adler, S. A. (1997). Information processing through the first year of life: A longitudinal study using the visual expectation paradigm. Monographs of the Society for Research in Child Development, i-160. https://doi.org/10.2307/1166196

Carpenter, M., Tomasello, M., \& Savage-Rumbaugh, S. (1995). Joint attention and imitative learning in children, chimpanzees, and enculturated chimpanzees. Social Development, 4(3), 217-237. https://doi.org/10.1111/j.1467-9507.1995.tb00063.x

Castiello, U. (2003). Understanding other people's actions: intention and attention. Journal of Experimental Psychology: Human Perception and Performance, 29(2), 416. https://doi.org/10.1037/0096-1523.29.2.416

Chawarska, K., \& Shic, F. (2009). Looking but not seeing: Atypical visual scanning and recognition of faces in 2 and 4-year-old children with autism spectrum disorder. Journal of Autism and Developmental Disorders, 39(12), 1663-1672. https://doi.org/10.1007/s10803-009-0803-7

Chawarska, K., Volkmar, F., \& Klin, A. (2010). Limited attentional bias for faces in toddlers with autism spectrum $\begin{array}{lllll}\text { disorders. Archives of } & \text { General }\end{array}$ https://doi.org/10.1001/archgenpsychiatry.2009.194

D'Entremont, B., \& Yazbek, A. (2007). Imitation of intentional and accidental actions by children with autism. Journal of Autism and Developmental Disorders, 37(9), 1665-1678. https://doi.org/10.1007/s10803-006-0291-y

Falck-Ytter T., Gredebäck G., \& von Hofsten C. (2006). Infants predict other people's action goals. Nature Neuroscience, 9(7), 878-879. https://doi.org/10.1038/nn1729

Falck-Ytter, T., Fernell, E., Gillberg, C., \& Von Hofsten, C. (2010). Face scanning distinguishes social from communication impairments in autism. Developmental Science, 13(6), 864-875. https://doi.org/10.1111/j.1467-7687.2009.00942.x

Flanagan, J. R., \& Johansson, R. S. (2003). Action plans used in action observation. Nature, 424(6950), 769-771. https://doi.org/10.1038/nature01861 
Frith, C. D., \& Frith, U. (2006). The neural basis of mentalizing. Neuron, 50(4), 531-534. https://doi.org/10.1016/j.neuron.2006.05.001

Gergely, G., Nádasdy, Z., Csibra, G., \& Bíró, S. (1995). Taking the intentional stance at 12 months of age. Cognition, 56(2), 165-193. https://doi.org/10.1016/0010-0277(95)00661-H

Gibson, J. J., \& Pick, A. D. (1963). Perception of another person's looking behavior. The American Journal of Psychology, 76(3), 386-394. https://doi.org/10.2307/1419779

Gredebäck, G., Johnson, S., \& von Hofsten, C. (2009). Eye tracking in infancy research. Developmental Neuropsychology, 35(1), 1-19. https://doi.org/10.1080/87565640903325758

Gredebäck, G., Stasiewicz, D., Falck-Ytter, T., Rosander, K., \& von Hofsten, C. (2009). Action type and goal type modulate goal-directed gaze shifts in 14-month-old infants. Developmental Psychology, 45(4), 1190. https://doi.org/10.1037/a0015667

Hamilton, A. F. D. C., Brindley, R. M., \& Frith, U. (2007). Imitation and action understanding in autistic spectrum disorders: how valid is the hypothesis of a deficit in the mirror neuron system? Neuropsychologia, 45(8), 1859-1868. https://doi.org/10.1016/j.neuropsychologia.2006.11.022

Hobson, J. A., \& Hobson, R. P. (2007). Identification: The missing link between joint attention and imitation? Development and Psychopathology, 19(2), 411-431. https://doi.org/10.1017/S0954579407070204

Kaiser, M. D., \& Pelphrey, K. A. (2012). Disrupted action perception in autism: behavioral evidence, neuroendophenotypes, and diagnostic utility. Developmental Cognitive Neuroscience, 2(1), 25-35. https://doi.org/10.1016/j.den.2011.05.005

Kasari, C., Gulsrud, A. C., Wong, C., Kwon, S., \& Locke, J. (2010). Randomized controlled caregiver mediated joint engagement intervention for toddlers with autism. Journal of Autism and Developmental Disorders, 40(9), 1045-1056. https://doi.org/10.1007/s10803-010-0955-5

Klin, A., Jones, W., Schultz, R., \& Volkmar, F. (2003). The enactive mind, or from actions to cognition: lessons from autism. Philosophical Transactions of the Royal Society of London B: Biological Sciences, 358(1430), 345-360. https://doi.org/10.1098/rstb.2002.1202

Klin, A., Lin, D. J., Gorrindo, P., Ramsay, G., \& Jones, W. (2009). Two-year-olds with autism orient to non-social contingencies rather than biological motion. Nature, 459(7244), 257-261. https://doi.org/10.1038/nature07868

Landa, R. J., Holman, K. C., \& Garrett-Mayer, E. (2007). Social and communication development in toddlers with early and later diagnosis of autism spectrum disorders. Archives of General Psychiatry, 64(7), 853-864. https://doi.org/10.1001/archpsyc.64.7.853

Landa, R. J., Holman, K. C., O'Neill, A. H., \& Stuart, E. A. (2011). Intervention targeting development of socially synchronous engagement in toddlers with autism spectrum disorder: a randomized controlled trial. Journal of Child Psychology and Psychiatry, 52(1), 13-21. https://doi.org/10.1111/j.1469-7610.2010.02288.x

Legerstee, M., Anderson, D., \& Schaffer, A. (1998). Five-and Eight-Month-Old Infants Recognize Their Faces and Voices as Familiar and Social Stimuli. Child Development, 69(1), 37-50. https://doi.org/10.1111/j.1467-8624.1998.tb06131.x

Lord, C., Rutter, M., DiLavore, P. C., \& Risi, S. (2002). Autism Diagnostic Observation Schedule. Western Psychological Services, Los Angeles.

Lord, C., Luyster, R., Gotham, K., \& Guthrie, W. J. (2012). Autism diagnostic observation schedule-toddler module. Los Angeles: Western Psychological Services.

Mahoney, G., \& MacDonald, J. (2007). Autism and developmental delays in young children: The Responsive Teaching curriculum for parents and professionals. Austin, TX: PRO-ED.

McPartland, J., Dawson, G., Webb, S. J., Panagiotides, H., \& Carver, L. J. (2004). Event-related brain potentials reveal anomalies in temporal processing of faces in autism spectrum disorder. Journal of Child Psychology and Psychiatry, 45(7), 1235-1245. https://doi.org/10.1111/j.1469-7610.2004.00318.x

Meltzoff, A. N., \& Brooks, R. (2001). "Like me" as a building block for understanding other minds: Bodily acts, attention, and intention. In B. F. Malle (Ed.), Intentions and intentionality: Foundations for social cognition (pp. 171-192). Cambridge, MA: MIT Press.

Mullen, E. M. (1995). Mullen scales of early learning (pp. 58-64). Circle Pines, MN: AGS. 
Mundy, P., \& Newell, L. (2007). Attention, joint attention, and social cognition. Current Directions in Psychological Science, 16(5), 269-274. https://doi.org/10.1111/j.1467-8721.2007.00518.x

Phillips, A. T., Wellman, H. M., \& Spelke, E. S. (2002). Infants' ability to connect gaze and emotional expression to intentional action. Cognition, 85(1), 53-78. https://doi.org/10.1016/S0010-0277(02)00073-2

Puce, A., Allison, T., Bentin, S., Gore, J. C., \& McCarthy, G. (1998). Temporal cortex activation in humans viewing eye and mouth movements. The Journal of Neuroscience, 18(6), 2188-2199. https://doi.org/10.1523/JNEUROSCI.18-06-02188.1998

Rogers, S. J., Hepburn, S. L., Stackhouse, T., \& Wehner, E. (2003). Imitation performance in toddlers with autism and those with other developmental disorders. Journal of Child Psychology and Psychiatry, 44(5), 763-781. https://doi.org/10.1111/1469-7610.00162

Rogers, S. J., Young, G. S., Cook, I., Giolzetti, A., \& Ozonoff, S. (2010). Imitating actions on objects in early-onset and regressive autism: Effects and implications of task characteristics on performance. Development and Psychopathology, 22, 71-85. https://doi.org/10.1017/S0954579409990277

Scaife, M., \& Bruner, J. S. (1975). The capacity for joint visual attention in the infant. Nature, 253(5489), 265-266. https://doi.org/10.1038/253265a0

Schafer, J. L. (1999). Multiple imputation: a primer. Statistical Methods in Medical Research, 8(1), 3-15. https://doi.org/10.1177/096228029900800102

Schreibman, L., Dawson, G., Stahmer, A. C., Landa, R., Rogers, S. J., McGee, G. G., ... \& McNerney, E. (2015). Naturalistic developmental behavioral interventions: Empirically validated treatments for autism spectrum disorder. Journal of Autism and Developmental Disorders, 45, 2411-2428. https://doi.org/10.1007/s10803-015-2407-8

Senju, A., Southgate, V., Miura, Y., Matsui, T., Hasegawa, T., Tojo, Y., ... \& Csibra, G. (2010). Absence of spontaneous action anticipation by false belief attribution in children with autism spectrum disorder. Development and Psychopathology, 22(02), 353-360. https://doi.org/10.1017/S0954579410000106

Servos, P., Osu, R., Santi, A., \& Kawato, M. (2002). The neural substrates of biological motion perception: an fMRI study. Cerebral Cortex, 12(7), 772-782. https://doi.org/10.1093/cercor/12.7.772

Striano, T., \& Reid, V. (2008). Social cognition at the crossroads: Perspectives on understanding others. In T. Striano \& V. M. Reid (Eds.), Social Cognition: Development, Neuroscience and Autism. Wiley-Blackwell Publishing.

Vivanti, G., Trembath, D., \& Dissanayake, C. (2014). Atypical monitoring and responsiveness to goal-directed gaze in autism spectrum disorder. Experimental Brain Research, 232(2), 695-701. https://doi.org/10.1007/s00221-013-3777-9

Vivanti, G., McCormick, C., Young, G. S., Abucayan, F., Hatt, N., Nadig, A., Ozonoff, S., \& Rogers, S. J. (2011). Intact and impaired mechanisms of action understanding in autism. Developmental Psychology, 47(3), 841. https://doi.org/10.1037/a0023105

von Hofsten, C., Uhlig, H., Adell, M., \& Kochukhova, O. (2009). How children with autism look at events. Research in Autism Spectrum Disorders, 3(2), 556-569. https://doi.org/10.1016/j.rasd.2008.12.003

Wetherby, A. M., Watt, N., Morgan, L., \& Shumway, S. (2007). Social communication profiles of children with autism spectrum disorders late in the second year of life. Journal of Autism and Developmental Disorders, 37, 960-975. https://doi.org/10.1007/s10803-006-0237-4

Woodward, A. L. (1998). Infants selectively encode the goal of an actor's reach. Cognition, 69, 1-34. https://doi.org/10.1016/S0010-0277(98)00058-4

Yu, C. \& Smith, L. B. (2017). Hand-eye coordination predicts joint attention. Child Development, 88(6), 2060-2078. https://doi.org/10.1111/cdev.12730

\section{Copyrights}

Copyright for this article is retained by the author(s), with first publication rights granted to the journal.

This is an open-access article distributed under the terms and conditions of the Creative Commons Attribution license (http://creativecommons.org/licenses/by/4.0/). 